\title{
Programmable Array Microscopes
}

Quentin Hanley, Rainer Heintzmann, Donna Arndt-Jovin, and Thomas Jovin

Max Planck Institute for Biophysical Chemistry, Göttingen, Germany

qhanley or tjovin @mpc186.mpibpc.gwdg.de.

The programmable array microscope (PAM) is a powerful tool combining the capabilities of nearly all previously described optical sectioning techniques in a single microscope. Not only can the user create optical sections of threedimensional objects, but the PAM's unique adaptive optical strategy allows a user to select the best sectioning method for a particular sample or experimental need. The key to the PAM is a spatial light modulator (SLM). This device, when placed in the image plane of a microscope, can be used to create optical sectioning, generate spatial encoding masks, and/or define regions of interest. The growing family of PAMs includes opti$\mathrm{cal}$ sectioning systems operating in fluorescence ${ }^{1,2}$ and reflection $^{3}$, and spectroscopic imaging systems for fluorescence emission spectroscopy ${ }^{4}$. Perhaps the most common applica-

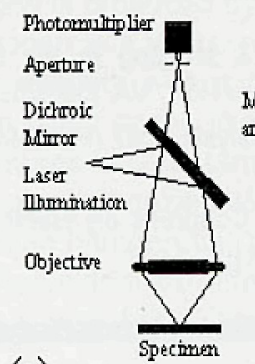

(a)

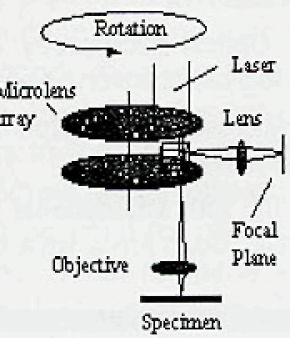

(b)

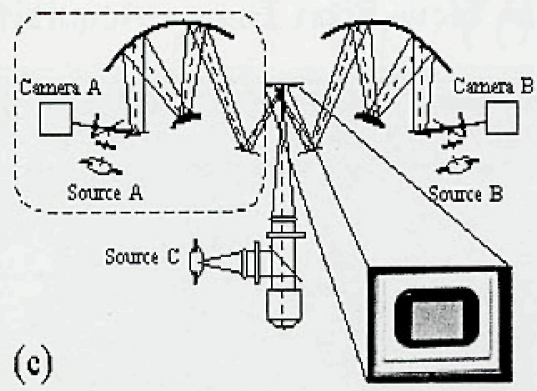

Figure 1 Confocal Scanning systems. The simplest and still most common optical sectioning microscope is the confocal laser scanning microscope (a). Here the scanning is done with the focused spot of a laser beam and out of focus light is removed at the confocal aperture. A variation on this approach is to use a line of illumination and slit detection. Another approach to confocal scanning is the use of regularly spaced apertures arranged on the surface of a rotating disk (b). This allows many apertures to scan the sample simultaneously. The system shown illustrates the use of microlenses to improve the utilization of illumination light. The disadvantage of the rotating disk is that the pattern of illumination and detection cannot be adjusted for a particular purpose. This problem is solved elegantly by the programmable array microscope (c). The PAM uses the elements of a spatial light modulator to scan a specimen. This allows the PAM to do point and line scans and to adapt the spacing of the pattern and the aperture size to a particular experimental need. The system shown illustrates acquisition of separate conjugate, $I_{c}$ ("in-focus"), and non-conjugate, $I_{n c}$ ("outof-focus"), images. When excitation source $A$ is activated, the "infocus" image forms on camera $A$ while an "out-of-focus" image forms on camera $\mathrm{B}$. Conversely, if excitation source $\mathrm{B}$ is activated, the positions of $I_{c}$ and $I_{n c}$ are reversed. The area surrounded by the dotted lines shows a single sided segment. The two-sided arrangement is currently under development. tion of this unique family of adaptive optical systems is optical sectioning.

Optical sectioning microscopes typically use single point, line, or rotating disk scanning (Figure 1). The rotating disk scanning systems normally consist of repeating patterns of dot or line arrays lithographically produced on the surface of a disk. Recently, disk systems utilising random bit sequences ${ }^{5}$ or regularly spaced apertures covered with microlenses have come into use. These modifications increase the optical throughput of the rotating disk and hence the speed of the measurement. Such microscopes have limitations when observing thick specimens and, since they are not made with adaptive components, are typically optimised for a particular magnification. The degree of optical sectioning depends on the periodic structure of the pattern, the numerical aperture of the objective, the wavelength of light, and the magnification. ${ }^{1,6}$ In this context, the programmable array microscope is advantageous since it allows most scanning methods to be reproduced under programmable control and the scan pattern can be adapted to best match a particular sample and lens.

The core of any programmable array microscope is a spatial light modulator placed in the primary image plane of the microscope. Spatial light modulators come in a variety of types and formats, with digital micromirror devices (DMDs) and liquid crystal devices (LCD) being the most common (Figure 2). Both technologies have been extensively developed to service the needs of video projector systems. As such, they are available in mass quantities at reasonable cost. The use of a DMD in optical sectioning arrangements provides the unique capability to select the nonconjugate (out-of-focus) light, which is normally lost at the pinhole in a confocal microscope, and sends it along a separate optical pathway (Figure 1c). ${ }^{6}$

A second application in which an adaptive optical element is useful is for generating spatial encoding masks for imaging spectroscopy. There are three standard ways in which twodimensional spectral images are acquired in microscopes. These consist of point, ${ }^{8}$ slit scanning, ${ }^{9}$ or wavelength scanning (Figure 3). ${ }^{10}$ These three methods cover the bulk of imaging spectroscopy done in microscopes. Wavelength scanning currently is the most popular of the methods shown and has been implemented with variable interference filters, acousto-optical tuneable filters, and liquid crystal tuneable filters. In situations in which only a limited area of the sample is of interest, or a limited number of wavelengths are needed, they remain the methods of choice. However, they share a common disadvantage in that the majority of the light is rejected or the majority of the sample is outside the region of view. When large areas of a sample are of interest, multiplexed

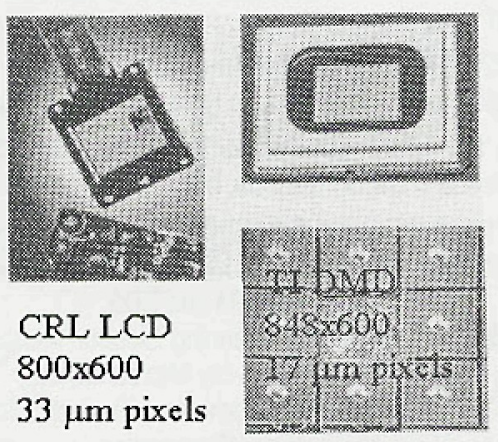

Figure 2 Two spatial light modulators used in PAMs. The SLM on the left is a transmission liquid crystal device provided by CRL laboratories. The SLM device on the right is a digital micromirror device (DMD) made by Texas Instruments. 


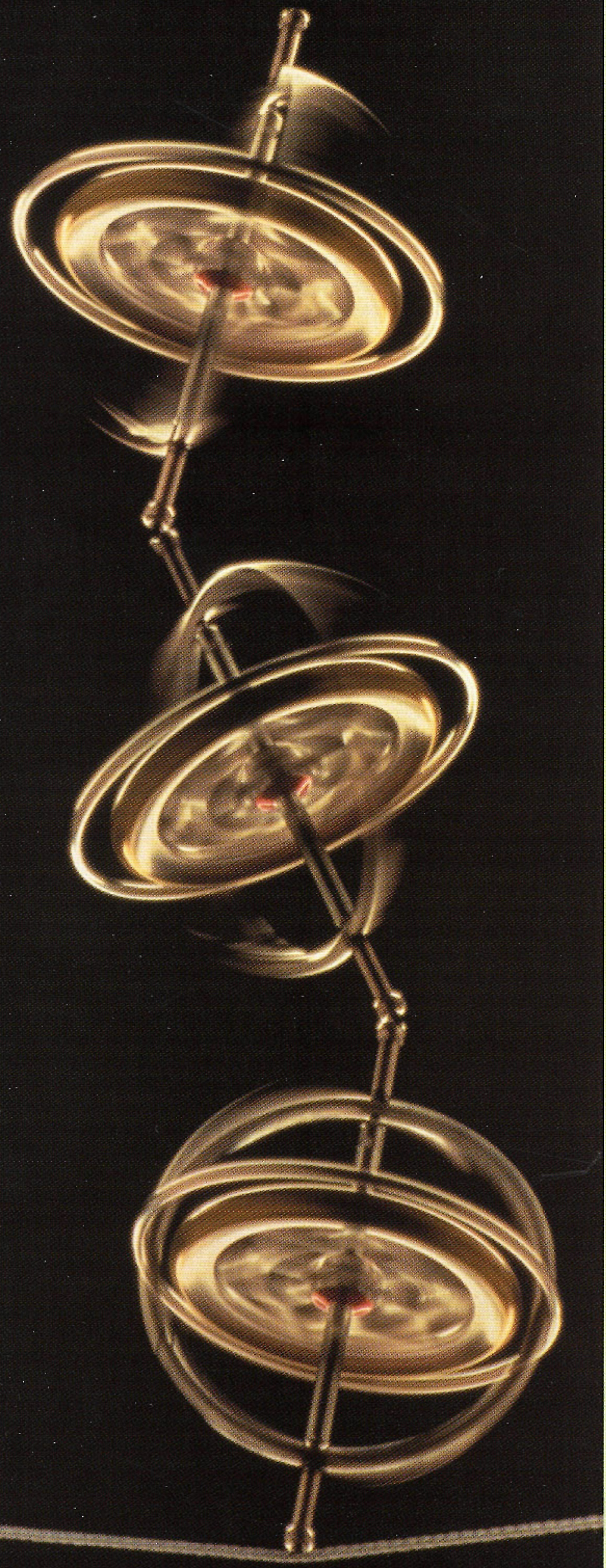

\section{Getting All Your Detectors} Working Together Can Be A Tough Balancing Act.

\section{That's Where Emispec Comes In.}

If balancing all components of your detectors has your head spinning, you should be talking to us.

You see, at Emispec Systems, Inc., we approach data acquisition differently. Instead of creating systems targeting one detector, we focus on integration. This concept can be applied equally to new and existing electron microscope installations. Core acquisition capabilities of our products include:

\section{- Digital scanning for STEM.}

- Digital EDX acquisition and analysis.

- EELS acquisition and analysis.

- CCD and TV imaging.

Integrated microscope control, imaging and spectroscopy allows automation of demanding experiments, such as spectrum imaging. Emispec enhances these capabilities with extensive on-line and off-line processing.

To find out how Emispec can help your lab keep in balance, visit our Web site today at www.emispec.com. See why we are fast becoming the leader in microscope detector technology solutions.

\section{Emispec}

Emispec Systems, Inc

2409 South Rural Road, Suite D

Tempe, Arizona 85282 USA

Phone: 480.894 .6443 - Fax: 480.894 .6458

Web: www.emispec.com

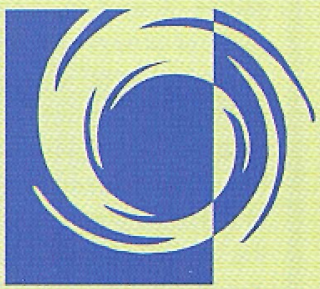

thinking beyond the box ${ }^{\mathrm{TM}}$ 


\section{Programmable Array Microscopes}

\section{Continued from page 8}

methods allow a greater amount of the sample to be observed simultaneously. Fourier encoding of the wavelength domain has proved successful for generating two-dimensional spectral images. ${ }^{11}$ Hadamard transform techniques, however, have a number of special advantages for this type of measurement. When implemented with a stationary mask technology, such as a liquid crystal (LC) SLM, it requires no moving parts. The optics are relatively simple and do not require exceptional care to align. Lithographic mask technologies, like the patterns used in rotating disks, cannot be rapidly adapted to meet the needs of a particular specimen or purpose under programmable control. The use of the spatial light modulator allows the element size to be adjusted for rapid screening at low resolution or careful examination of higher resolution structures.

\section{Optical Sectioning Programmable Array Microscopes}

The optical sectioning PAM was implemented as an addon module to a Nikon E-600 microscope. A Texas Instruments DMD (Plano, TX) was mounted in the primary image plane and used to define patterns of illumination and detection. The light from the "on" elements was relayed to an Apogee KX-2 charge-coupled device (CCD) camera (Apogee Instruments, Tucson, AZ). The quality of the optical relay was sufficient to observe effects from the $1 \mu \mathrm{m}$ dark segments between adja-

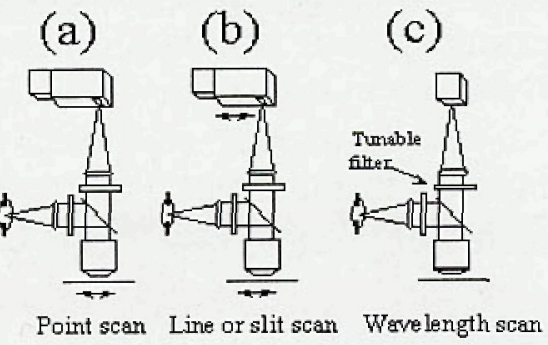

$$
\text { (d) }
$$

(e)

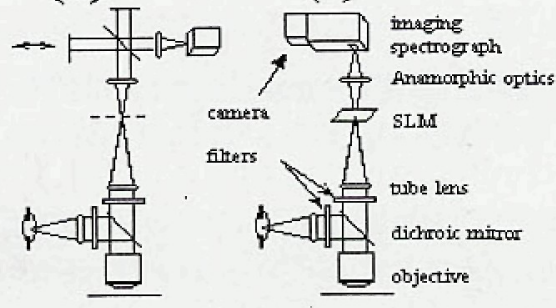

Fourier encoding Hadamard encoding

Figure 3 Spectroscopic imaging methods. The most common are point (a), line (b), and wavelength scanning (c). Point scanning systems look at a single point in the image field and record the spectrum after passing the light through a scanning monochromator or spectrograph. Observing line transects can increase the speed of acquisition of the point scanning approach. In the latter approach, a camera is used to detect a two dimensional image consisting of one spatial axis and wavelength. The two dimensional spectral image is then acquired by recording a series of such images at different spatial locations. For imaging a small number of wavelengths, the use of a wavelength tunable filter and CCD camera detection is one of the most efficient. These methods share a common disadvantage in that most of the sample or the range of available wavelengths is not available for observation. To avoid these limitations, multiplexed methods have been developed using both Fourier (d) and Hadamard encoding techniques (e). The latter is well adapted to the PAM approach. With a transmissive liquid crystal SLM there are no moving parts, a low demand on detector dynamic range, and the high tolerances required for interferometry are not present. cent DMD elements. The DMD was illuminated through a Nikon filter block using a $250 \mathrm{~W}$ super high pressure $\mathrm{Hg}$ arc lamp system (Lumatec GmbH, Munich, Germany), a 450 W Xe arc lamp (Müller Elektronik-Optik, Moosinning, Germany), or an argon ion laser (Coherent, Santa Clara, CA). Axial positioning of the objective was accomplished using a PIFOC piezoelectric system with a resolution of $10 \mathrm{~nm}$ (Physik Instrumente, Waldbronn, Germany). A block diagram of the microscope and add-on is given in figure $1 \mathrm{c}$.

\section{Spectroscopic PAM}

The fluorescence Hadamard transform programmable array microscope (PAM) was as shown in Figure $3 e^{7}$ The system consisted of an add-on to a Nikon E-600 microscope equipped with a $100 \mathrm{~W} \mathrm{Hg}$ arc lamp and an epi-fluorescence unit. This spectroscopy module consisted of a SVGA format LCD device SLM (Central Research Labs, England), anamorphic relay optics, a PARISS prism-based imaging spectrograph (Lightform Inc., Belle Mead, NJ), and a KX-2 CCD camera (Apogee Instruments, Tucson, AZ). The pixels of the SLM consisted of an irregular element approximating a $26 \mu \mathrm{m}$ (horizontal) by $24 \mu \mathrm{m}$ (vertical) rectangle reproduced on a $33 \mu \mathrm{m}$ square pitch. The active element was a TFT twisted nematic LC array in an $800 \times 600$ format. The SLM was used to display a series of bar patterns defined by the rows of cyclic S-matrices. An image was recorded for every row of the Smatrix. After subsequent decoding with an inverse S-transform, a three-dimensional image stack was obtained.

\section{Axial responses}

Results:

When used to collect conjugate images, measured axial responses to rhodamine thin films indicated a resolution of $\sim 1.0 \mu \mathrm{m}$ for line patterns having a $1 \times 24$ unit cell and $\sim 0.6 \mu \mathrm{m}$ for the dot lattices having a unit cell of $4 \times 6$. A conventional illumination pattern could not discriminate the axial location of the thin fluorescent film. The offsets in these axial responses were characterized in terms of $\kappa$, the ratio of "on" to total modulator elements, and $\kappa$ ', the ratio of axial response maximum to minimum. Both patterns had $\kappa=0.041$, but the line patterns had $\kappa^{\prime}=0.18$ while the dot lattices had $\kappa^{\prime}=0.38$. This implies that a line pattern will show less background than a dot pattern for equivalent $\kappa$ while giving a thicker optical section. In addition we found that for the same throughput, a random pattern has a lower offset than has a regular pattern of lines or dots.
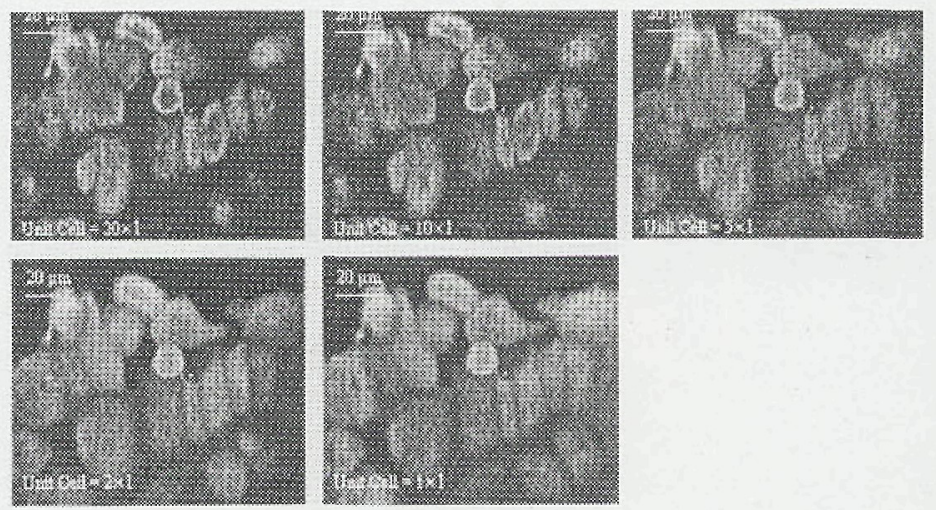

Figure 4. Images of MCF-7 cells stained for tubulin with monoclonal antibody and Oregon green GAMIG, 60X NA 1.4 oil immersion objective. This image shows a comparison of different scanning strategies implemented in the optically sectioning programmable array microsope. While the best sectioning is obtained using the largest unit cell $(20 \times 1)$, there is relatively little loss of quality for the $10 \mathrm{X}$ unit cell. The latter can be scanned twice as fast. 

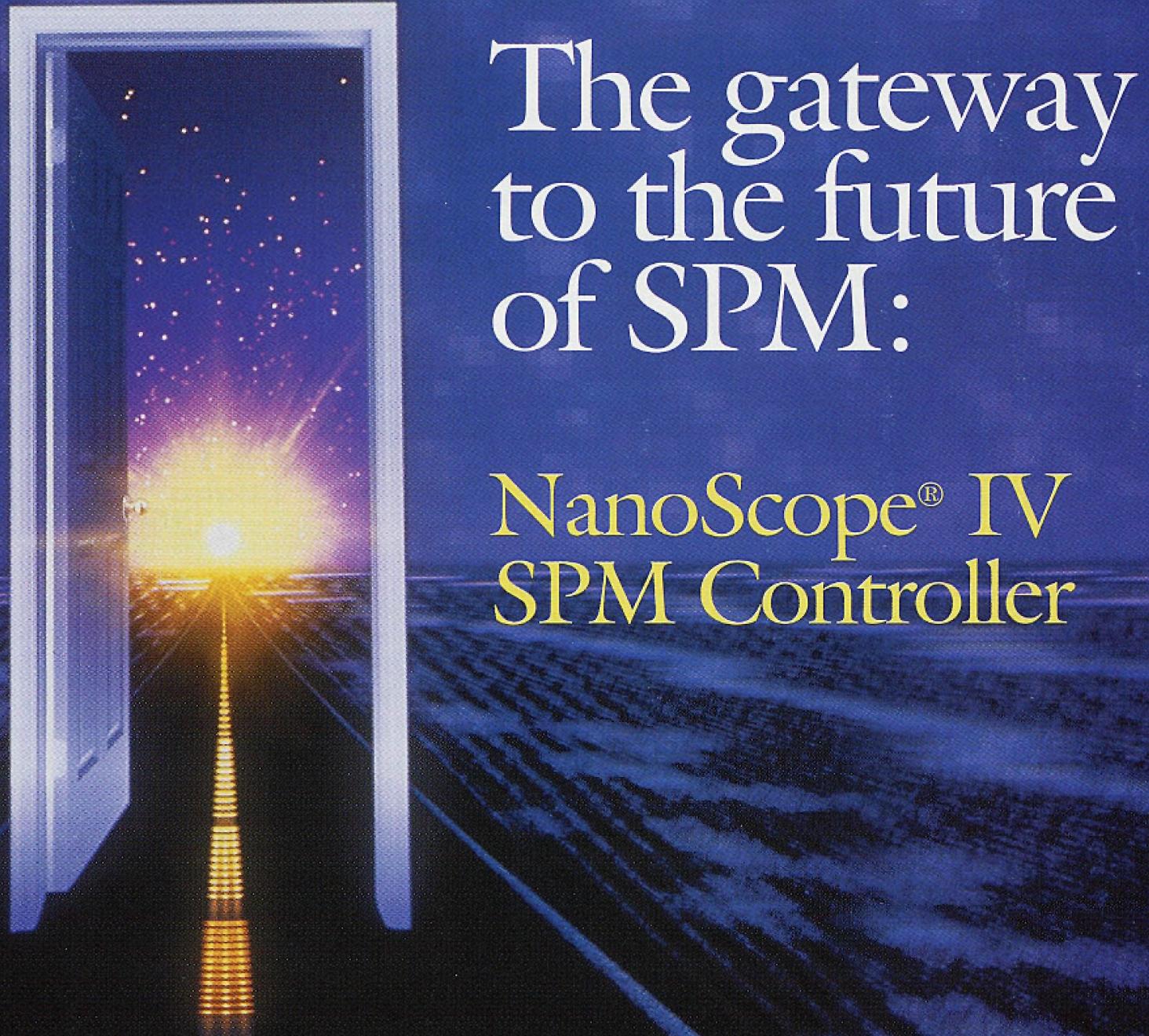

\section{NanoScope ${ }^{\oplus}$ IV SPM Controller}

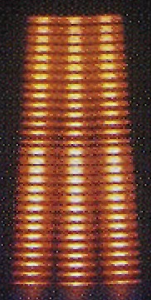

The interface you need for expanding scanning probe microscope capabilities.

- More speed

- More resolution

- More functionality

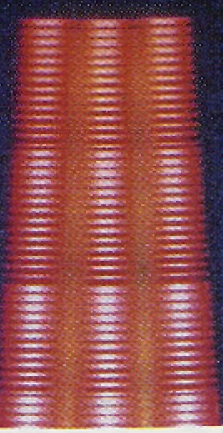

- More flexibility

To find out more, go to www.di.com or call 1.800 .873 .9750 . 


\section{Programmable Array Microscopes \\ Continued from page 10}

\section{Observation of biological specimens}

The sectioning capability seen with the thin films was also observed in biological specimens (Figure 4). As the spacing of the illumination/detection pattern decreased, the amount of blur from out-of-focus planes increased, as does the amount of cross talk between adjacent apertures in the pattern. Relatively little degradation in image quality is seen between the $20 \times 1$ and the $10 \times 1$ patterns in the specimen shown. The latter pattern, however, can be scanned twice as fast.

\section{Spectroscopic imaging}

Stacks of three-dimensional spectroscopic images can be collected using the spectroscopic system (Figure 5) generating a spectroscopic image with 3 spatial dimensions. Similar observations of polytene chromosomes from Drosophila melanogaster stained with Alexa594 or Cy3 for the polyhomeotic protein and with YOYO-1 for DNA revealed two spectral signatures, one represented predominantly the fluorescence emission from YOYO-1/DNA and the other from $\mathrm{Cy} 3$ or $\mathrm{Al}$ exa594. From the four-dimensional data set obtained from observation of Drosophila melanogaster, two three-dimensional wavelength slices were extracted and submitted to deconvolution analysis (figure 6). This procedure allows much of the out of focus blur to be removed giving a better indication of important proteins along the chromosome backbone.

\section{Conclusions}

The PAM uniquely combines the power of multiple scanning methods in a single microscope, allowing the results to be compared in a rapid and convenient manner. For any given sample, the selection of an optical sectioning strategy represents a compromise between light throughput, axial resolution, offset rejection, and photobleaching. Deciding which method to use will depend on the sample, the needs of the investigator, and many other experimental variables. The PAM can rapidly adapt its sectioning strategy to best match a particular specimen. In addition, the PAM is a powerful platform on which to develop spectroscopic imaging methods. In both optical sectioning and imaging spectroscopy, the adaptive capacity of the spatial light modulator gives an unparalleled flexibility to the system. Hybrid PAMs combining optical sectioning with emis-
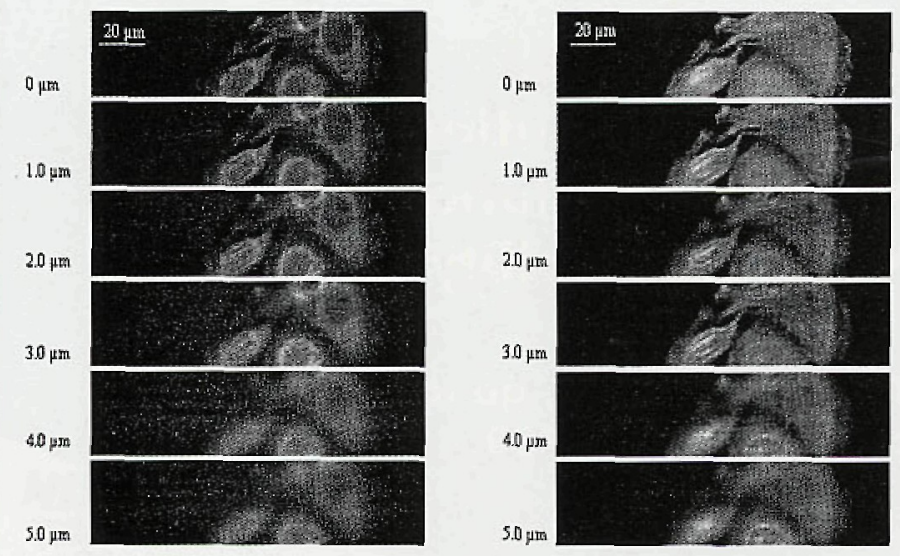

Figure 5 Series of two-dimensional spectral images taken at $1 \mu \mathrm{m}$ along the $z$-axis. Each series represents one wavelength slice in the data set. The image series on the left corresponds to approximately $630 \mathrm{~nm}$ (MitoTracker). The series on the right corresponds to approximately 525 $\mathrm{nm}$ (Oregon Green). sion spectroscopy and lifetime imaging are under development and the two sided arrangement shown in Figure $1 \mathrm{C}$ has recently been realized.

1. Q. S. Hanley, P. J. Verveer, M. J. Gemkow, D. Arndt-Jovin and T. M. Jovin. J. Microsc. 196, 317 (1999).

2. Q. S. Hanley, P. J. Verveer and T. M. Jovin. Appl. Spectrosc. 52, 783 (1998).

3. S. D. Cha, P. C. Lin, L. J. Zhu, P. C. Sun and Y. Fainman. Appl. Opt. 39, 2605 (2000).

4. Q. S. Hanley, P. J. Verveer, D. J. Arndt-Jovin and T. M. Jovin. J. Microsc. 197, 5 (2000).

5. R. Juskaitis, T. Wilson, M. A. A. Neil and M. Kosubek. Nature 383, 804 (1996).

6. P. J. Verveer, Q. S. Hanley, P. W. Verbeek, L. J. Van Vliet and T. M. Jovin. J. Microsc. 189, 192 (1998).

7. Q. S. Hanley, P. J. Verveer and T. M. Jovin. Appl. Spectrosc. 53, 1 (1999).

8. G. J. Puppels, F. F. M. de Mul, C. Otto, J. Greve, M. RobertNicoud, D. J. Arndt-Jovin and T. M. Jovin. Nature 347, 301 (1990).

9. J. A. Timlin, A. Carden, M. D. Morris, J. A. Bonadio, C. E. Hoffler, K. M. Kozloff and S. A. Goldstein. J. Biomed. Opt. 4, 28 (1999).

10. M. D. Schaeberle, H. R. Morris, J. F. Turner and P. J. Treado. Anal. Chem. 5, 175A (1999).

11. Z. Malik, R. A. Buckwald, A. Talmi, Y. Garini and S. G. Lipson. J. Microsc. 182, 133 (1996).
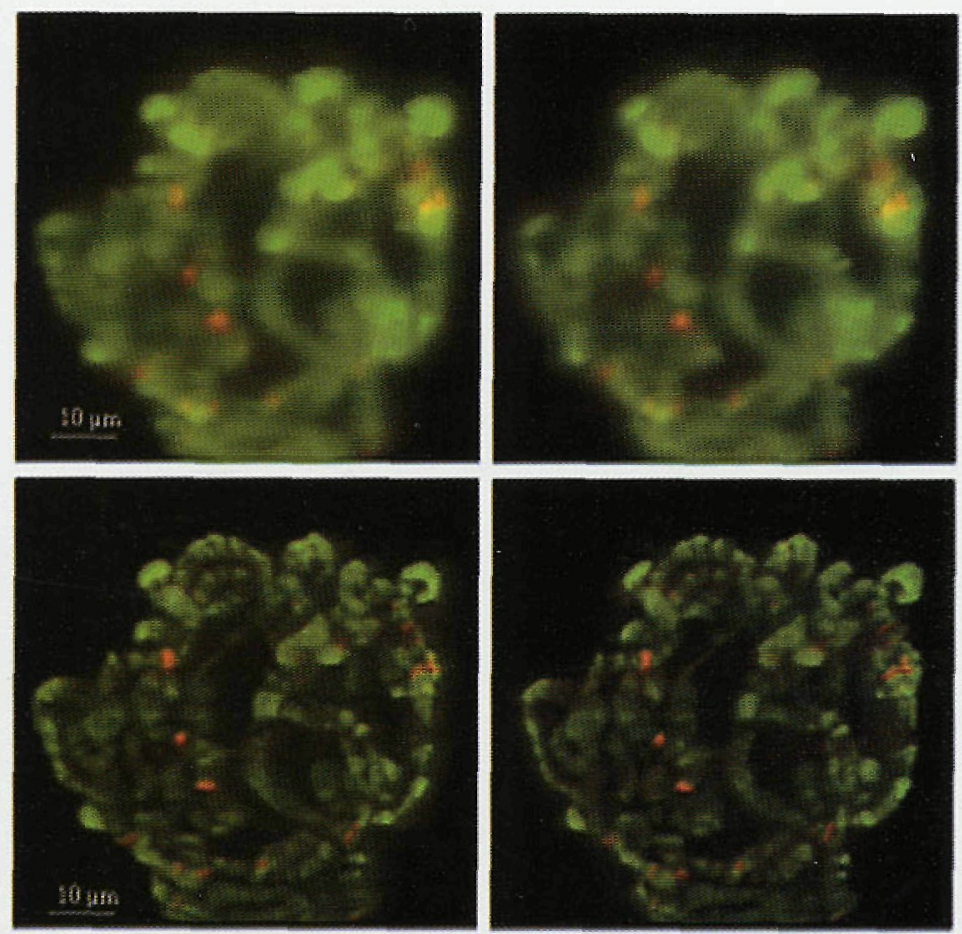

Figure 6 Stereo projection representing two spectral segments of a threedimensional spectroscopic image before (upper panel) and after (lower panel) image reconstruction. These images show Drosophila polytene chromosomes stained for the polyhomeotic protein with Alexa598 and DNA with YOYO-1. Recontruction is from a stack of 16 spectral images measured at $400 \mathrm{~nm}$ intervals in the axial direction. (Data reprinted with permission of the Journal of Microscopy 197:5-14 (2000)). 
